\title{
Goos-Hänchen Lateral Displacements at the Interface between Isotropic and Gyroelectric Media
}

\author{
Jinbao Wang, ${ }^{1}$ Hui Huang, ${ }^{1}$ Cheng Chen, ${ }^{1}$ Hanfeng He, ${ }^{2}$ Yuanyuan Dong, ${ }^{1}$ and Huan $\mathbf{Q i}^{1}$ \\ ${ }^{1}$ School of Electrical Engineering, Beijing Jiaotong University, Beijing 100044, China \\ ${ }^{2}$ Sichuan Electric Power Corporation, Chengdu 610041, China \\ Correspondence should be addressed to Hui Huang; hhuang@bjtu.edu.cn
}

Received 1 March 2013; Revised 15 May 2013; Accepted 29 May 2013

Academic Editor: Tat Yeo

Copyright (c) 2013 Jinbao Wang et al. This is an open access article distributed under the Creative Commons Attribution License, which permits unrestricted use, distribution, and reproduction in any medium, provided the original work is properly cited.

\begin{abstract}
A detailed study on Goos-Hänchen $(\mathrm{GH})$ lateral displacements of the reflected and transmitted waves propagating at the interface between an isotropic medium and a gyroelectric medium in Voigt configuration is presented. After the reflection coefficient and transmission coefficient are derived, based on the stationary phase approach, GH lateral displacements are obtained analytically. The numerical results for a specific gyroelectric medium are also given. It shows that with the existence of an applied magnetic field, the $\mathrm{GH}$ effect occurs not only during total reflection but also during nontotal reflection, which is not true for isotropic media. Moreover, due to the nonreciprocal property of the gyroelectric medium, the sign of the incident angle also influences the displacements. Finite-element method simulations have verified the theoretical results.
\end{abstract}

\section{Introduction}

Gyroelectric medium is an electron plasma with an applied magnetic field. The characteristics of electromagnetic waves propagation in gyroelectric plasmas have been theoretically investigated in many literatures. The magnetoplasma modes in Voigt, perpendicular, and Faraday configurations have been studied by Kushwaha and Halevi [1-3]; Gillies and Hlawiczka have done some researches on gyrotropic waveguides in detail [4-8], and dyadic Green's functions for gyrotropic medium have been investigated by both Eroglu and Li [9-11]. There are also some studies focusing upon the effects of magnetic field on semiconducting plasma slab and negatively refracting surfaces $[12,13]$. Furthermore, propagation and scattering characteristics in gyrotropic systems $[9,14]$ and surface modes at the interface of a special gyroelectric medium [15] have been investigated extensively.

The Goos-Hänchen (GH) effect $[16,17]$ has been studied for many years. GH lateral displacement refers to the spatial displacement of a reflected wave from the position expected by geometrical reflection. Traditionally, GH shift is always a phenomenon during total reflection, when an electromagnetic beam is reflected at the interface between media with different reflective indexes. The lateral displacement has the well-known expression $S=-\mathrm{d} \phi / \mathrm{d} k_{y}$, which was proposed by Artmann using the stationary phase method [18]. The GH effect has been analyzed both theoretically $[19,20]$ and experimentally [21-23]. The lateral shifts were found to be positively or negatively large for both reflected and transmitted beams in different media such as dielectric surfaces or slabs [24-26], metal surfaces [27], dielectric-chiral surface [28-31], absorptive media [32-34], and metamaterial [35-38].

In this paper, we focus on the reflection and transmission characteristics of an electromagnetic beam propagating at the interface between an isotropic medium and a gyroelectric medium in Voigt configuration. After obtaining the analytical expressions for the reflection and transmission coefficients, we get the mathematical result for the GH lateral displacement by the stationary phase approach. Based on the results, we discuss some unique phenomena.

\section{Reflection, Transmission, and Goos-Hänchen Lateral Displacements}

Considering the configuration in Figure 1, a plane wave is incident from a semi-infinite isotropic medium into a gyroelectric medium at an oblique angle $\theta_{i}$ with respect to 


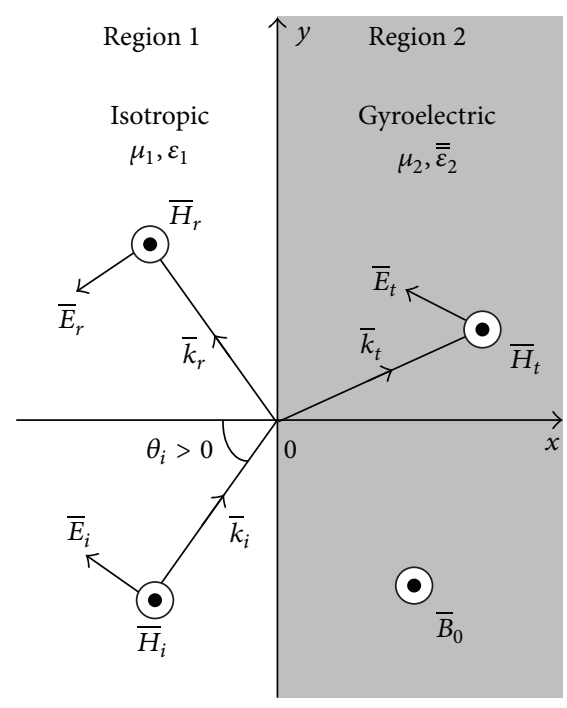

FIGURE 1: Reflection and transmission of TM waves at an interface between a semi-infinite isotropic medium (region 1) and a gyroelectric medium in the Voigt configuration (region 2). In region 2, applied magnetic field $\bar{B}_{0}$ is in $+z$ direction.

the normal of the interface. Region 1 is an isotropic medium, with permeability $\mu_{1}$ and permittivity $\varepsilon_{1}$. In region 2 , an external magnetic field $\bar{B}_{0}$ is applied in $+z$ direction, parallel to the interface and perpendicular to the direction of the wave propagation (i.e., gyroelectric medium in Voigt configuration). Its permeability is $\mu_{2}$, while permittivity $\overline{\overline{\varepsilon_{2}}}$ is a tensor, which takes the following form:

$$
\overline{\bar{\varepsilon}}_{2}=\left[\begin{array}{ccc}
\varepsilon_{x x} & i \varepsilon_{g} & 0 \\
-i \varepsilon_{g} & \varepsilon_{y y} & 0 \\
0 & 0 & \varepsilon_{z z}
\end{array}\right],
$$

where the matrix elements are given by

$$
\begin{gathered}
\varepsilon_{x x}=\varepsilon_{y y}=\varepsilon_{\infty}\left(1-\frac{\omega_{p}^{2}}{\omega^{2}-\omega_{c}^{2}}\right), \\
\varepsilon_{z z}=\varepsilon_{\infty}\left(1-\frac{\omega_{p}^{2}}{\omega^{2}}\right), \\
\varepsilon_{g}=\varepsilon_{\infty}\left[-\frac{\omega_{p}^{2} \omega_{c}}{\omega\left(\omega^{2}-\omega_{c}^{2}\right)}\right] .
\end{gathered}
$$

Here, $\omega_{p}=\sqrt{N q_{e}^{2} / m_{\mathrm{eff}} \varepsilon_{\infty}}$ and $\bar{\omega}_{c}=q_{e} \bar{B}_{0} / m_{\mathrm{eff}}$ are the plasma and cyclotron frequencies, respectively, $\varepsilon_{\infty}$ is the background permittivity, $N$ is the electron density, $m_{\text {eff }}$ is the effective mass, and $q_{e}$ is the electron charge.

It is known that for gyroelectric medium in the Voigt configuration, waves can be decoupled into TE and TM modes, and only the TM mode is affected by the gyrotropy $[13,14]$. Thus, we focus on the TM case.

For Figure 1, with wave vectors $\bar{k}_{i}=\hat{y} k_{y}+\widehat{x} k_{1 x}$ (incident) and $\bar{k}_{r}=\hat{y} k_{y}-\hat{x} k_{1 x}$ (reflection) in the isotropic medium, $\bar{k}_{t}=\hat{y} k_{y}+\hat{x} k_{2 x}$ (transmission) in the gyroelectric medium, the dispersion relations can be expressed as $[13,14]$

$$
\begin{aligned}
& k_{y}^{2}+k_{1 x}^{2}=\omega^{2} \mu_{1} \varepsilon_{1}, \\
& k_{y}^{2}+k_{2 x}^{2}=\omega^{2} \mu_{2} \varepsilon_{V} .
\end{aligned}
$$

Here, $\varepsilon_{V}=\left(\varepsilon_{x x}^{2}-\varepsilon_{g}^{2}\right) / \varepsilon_{x x}$ is the equivalent permittivity of the gyroelectric medium in the Voigt configuration for TM waves.

2.1. Nontotal Reflection Case. According to the Maxwell equations and boundary conditions, when there is no total reflection at the boundary, the transmission and reflection coefficients for TM waves can be written as

$$
\begin{aligned}
& R=\frac{\sigma k_{1 x}-k_{2 x}+i \tau k_{y}}{\sigma k_{1 x}+k_{2 x}-i \tau k_{y}}, \\
& T=\frac{2 \sigma k_{1 x}}{\sigma k_{1 x}+k_{2 x}-i \tau k_{y}},
\end{aligned}
$$

where the parameters $\sigma$ and $\tau$ are both dimensionless, defined as

$$
\sigma=\frac{\varepsilon_{V}}{\varepsilon_{1}}, \quad \tau=\frac{\varepsilon_{g}}{\varepsilon_{x x}} .
$$

According to (5), both reflection coefficient $R$ and transmission coefficient $T$ are not real. They always have an imaginary part due to $\tau$ as a result of the gyrotropy. Hence, the $\mathrm{GH}$ angle $\phi$ of either reflection or transmission is not zero. Therefore, there always exists a GH lateral displacement, although there is no total reflection at the boundary.

Noting that the denominators of both coefficients are the same, the GH lateral displacements of the reflection and transmission for TM waves can be expressed as

$$
\begin{aligned}
S_{t}= & -\frac{1}{|T|^{2}}\left\{\operatorname{Re}[T] \frac{\partial \operatorname{Im}[T]}{\partial k_{y}}-\operatorname{Im}[T] \frac{\partial \operatorname{Re}[T]}{\partial k_{y}}\right\} \\
= & \frac{-\tau k_{y}}{\left(\sigma k_{1 x}+k_{2 x}\right)^{2}+\left(\tau k_{y}\right)^{2}} \\
& \times\left(\frac{\sigma k_{1 x}+k_{2 x}}{k_{y}}+\frac{\sigma k_{y}}{k_{1 x}}+\frac{k_{y}}{k_{2 x}}\right), \\
S_{r}= & -\frac{1}{|R|^{2}}\left\{\operatorname{Re}[R] \frac{\partial \operatorname{Im}[R]}{\partial k_{y}}-\operatorname{Im}[R] \frac{\partial \operatorname{Re}[R]}{\partial k_{y}}\right\} \\
= & S_{t}+\Delta S_{r t},
\end{aligned}
$$

where

$$
\begin{aligned}
\Delta S_{r t}= & \frac{-\tau k_{y}}{\left(\sigma k_{1 x}-k_{2 x}\right)^{2}+\left(\tau k_{y}\right)^{2}} \\
& \times\left(\frac{\sigma k_{1 x}-k_{2 x}}{k_{y}}+\frac{\sigma k_{y}}{k_{1 x}}-\frac{k_{y}}{k_{2 x}}\right) .
\end{aligned}
$$




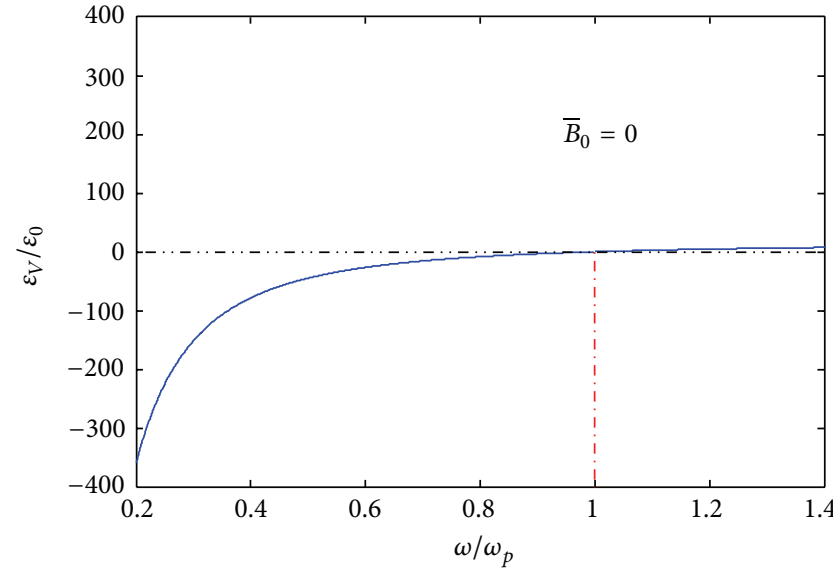

(a)

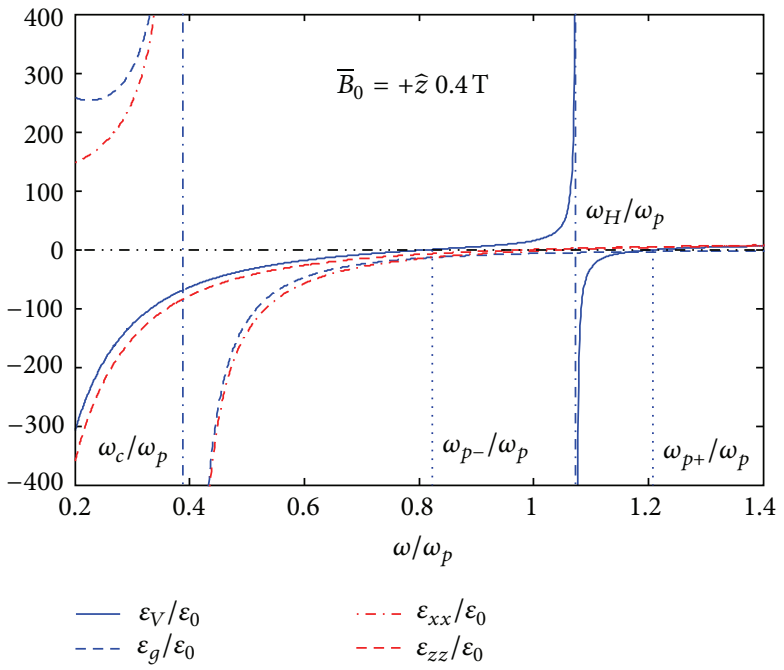

(b)

FIGURE 2: The frequency dependence of the permittivity tensor and the corresponding equivalent permittivity $\varepsilon_{V}$ for gyroelectric medium (in the Voigt configuration). The external magnetic field is (a) $B_{0}=0$ and (b) $\bar{B}_{0}=+\widehat{z} 0.4 \mathrm{~T}$, respectively.

It is interesting to note that both $S_{t}$ and $S_{r}$ are proportional to $\tau$, the unique parameter of the gyroelectric medium, and $\tau$ is a direct manifestation of the applied magnetic field $\bar{B}_{0}$ (see (6)). In fact, if the external magnetic field is zero, that is, $\omega_{c}=$ 0 , according to the permittivity tensor (see (1) and (2a), (2b), and $(2 c)$ ), it is reasonable to consider the gyroelectric medium as a dispersive isotropic medium. Furthermore, without the external magnetic field, both $\varepsilon_{g}$ and $\tau$ are zero, and according to (7)-(8), the GH lateral displacements are also zero, which are consistent with the fact that GH displacement is always a phenomenon along with total reflection for isotropic media case.

2.2. Total Reflection Case. When $k_{y}^{2}>\omega^{2} \mu_{2} \varepsilon_{V}$, according to (4), $k_{2 x}$ becomes imaginary, that is, total reflection occurs at the boundary. Let $k_{2 x}=i \alpha$, the dispersion relation of gyroelectric medium becomes $k_{y}^{2}-\alpha^{2}=\omega^{2} \mu_{2} \varepsilon_{V}$, and (5) can be rewritten as

$$
\begin{aligned}
& R^{\prime}=\frac{\sigma k_{1 x}-i \alpha+i \tau k_{y}}{\sigma k_{1 x}+i \alpha-i \tau k_{y}}, \\
& T^{\prime}=\frac{2 \sigma k_{1 x}}{\sigma k_{1 x}+i \alpha-i \tau k_{y}} .
\end{aligned}
$$

Since the transmitted wave is evanescent in the $x$ direction, our focus is only upon the GH lateral displacement of the reflection, which can be expressed as

$$
\begin{aligned}
S_{r}^{\prime} & =-\frac{1}{\left|R^{\prime}\right|^{2}}\left\{\operatorname{Re}\left[R^{\prime}\right] \frac{\partial \operatorname{Im}\left[R^{\prime}\right]}{\partial k_{y}}-\operatorname{Im}\left[R^{\prime}\right] \frac{\partial \operatorname{Re}\left[R^{\prime}\right]}{\partial k_{y}}\right\} \\
& =\frac{2 \sigma k_{1 x}}{\left(\sigma k_{1 x}\right)^{2}+\left(\alpha-\tau k_{y}\right)^{2}}\left[\frac{k_{y}\left(\alpha-\tau k_{y}\right)}{k_{1 x}^{2}}+\frac{k_{y}}{\alpha}-\tau\right] .
\end{aligned}
$$

We can see that if total reflection occurs, the lateral displacement $S_{r}^{\prime}$ is not zero, even for the case without applied magnetic field $(\tau=0)$. It means that for isotropic media, there is $\mathrm{GH}$ effect during the total reflection, which is the fact we have known.

\section{Result and Discussion}

Here, we consider an indium antimony (InSb) with an external magnetic field as the gyroelectric medium. The isotropic medium is a vacuum. The material parameters used in the computation are $\mu_{1}=\mu_{2}=\mu_{0}, \varepsilon_{1}=\varepsilon_{0}, \varepsilon_{\infty}=15 \varepsilon_{0}, N=$ $10^{22} \mathrm{~m}^{-3}$, and $m_{\text {eff }}=0.015 m_{0}=0.13664 \times 10^{-31} \mathrm{~kg}$ [39-41]. Hence, $\omega_{p}=1.19 \times 10^{13} \mathrm{rad} / \mathrm{s}$ and $\omega_{c} / \omega_{p}=0.98 B_{0}$.

For TM waves, since $\varepsilon_{x x}$ and $\varepsilon_{g}$ change with frequency, the equivalent permittivity $\varepsilon_{V}$ is also dependent on frequency, as shown in Figure 2. The applied magnetic field $B_{0}$ splits $\varepsilon_{x x}$ into 2 branches with the boundary of cyclotron frequency $\omega_{c}$, and the case of $\varepsilon_{g}$ is similar. Thus, the equivalent permittivity $\varepsilon_{V}$ also has 2 branches with another boundary of $\omega_{H}$, and each branch has its own plasma frequency, denoted by $\omega_{p-}$ and $\omega_{p^{+}}$, shown as [42]

$$
\begin{gathered}
\omega_{H}=\sqrt{\omega_{c}^{2}+\omega_{p}^{2}}, \\
\omega_{p \pm}=\frac{1}{2}\left( \pm \omega_{c}+\sqrt{\omega_{c}^{2}+4 \omega_{p}^{2}}\right) .
\end{gathered}
$$

We plot the GH lateral displacements in the $\left(\omega, \theta_{i}\right)$ plane with a color scale proportional to the magnitude, as shown in Figure 3 . The horizontal axis is the frequency, normalized to the plasma frequency $\omega_{p}$, and the vertical axis is the incident angle, from $-85^{\circ}$ to $+85^{\circ}$. We define the angle to be positive 


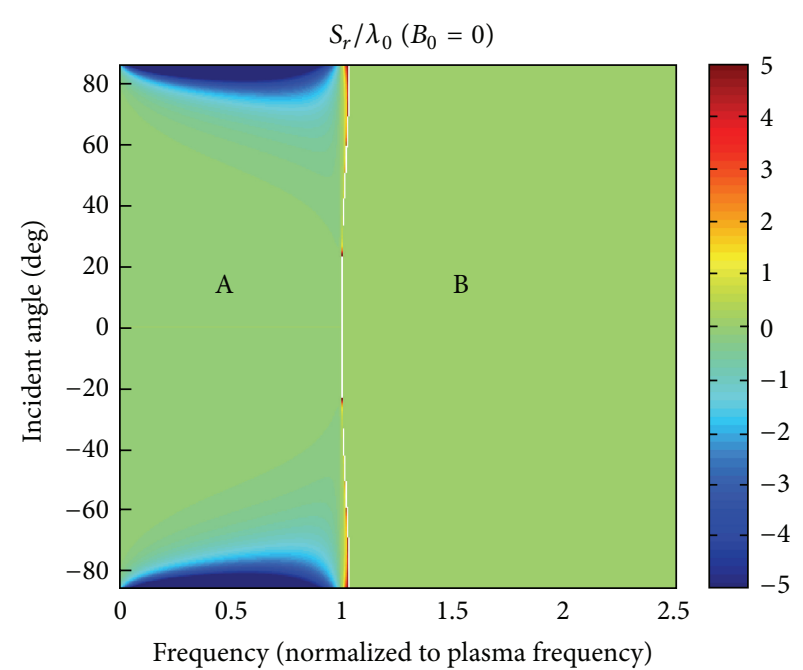

(a)

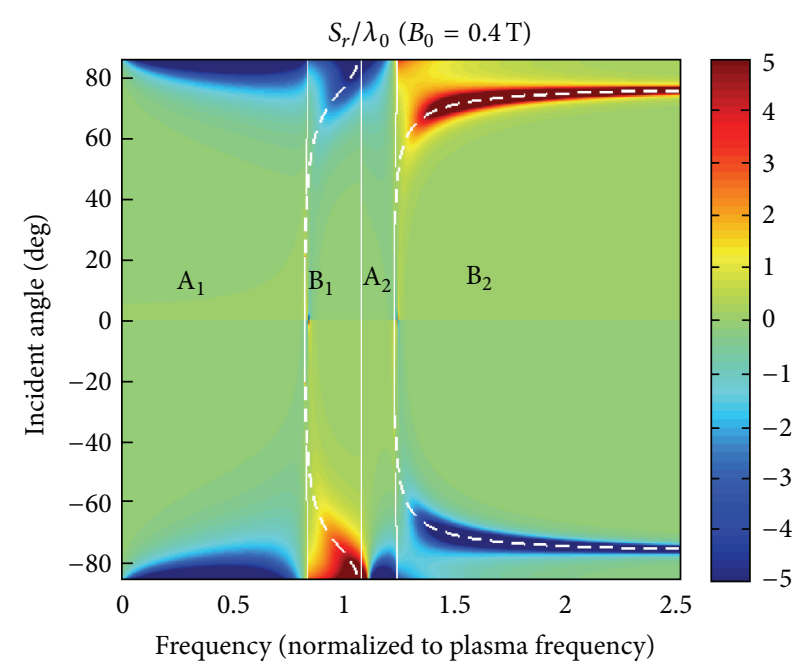

(c)

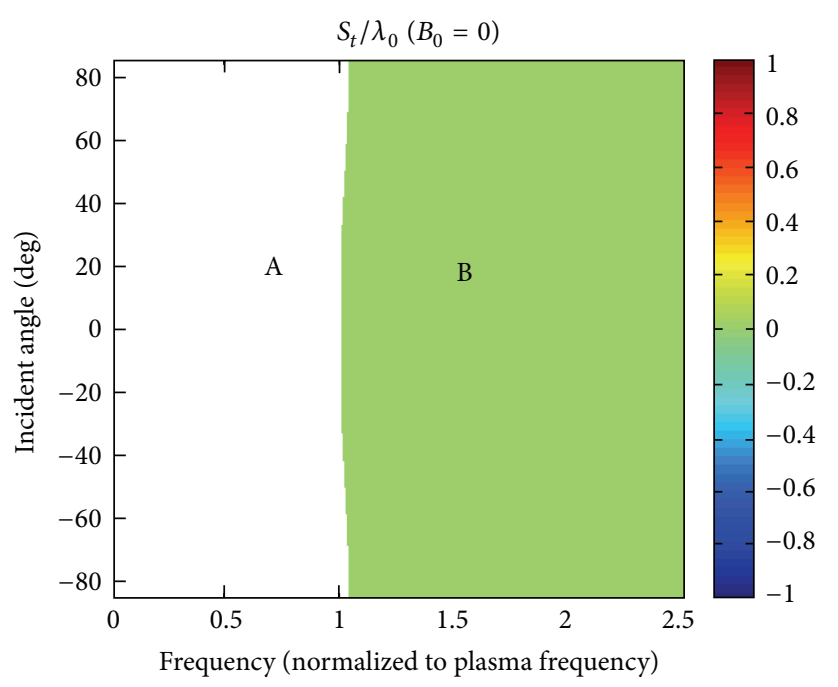

(b)

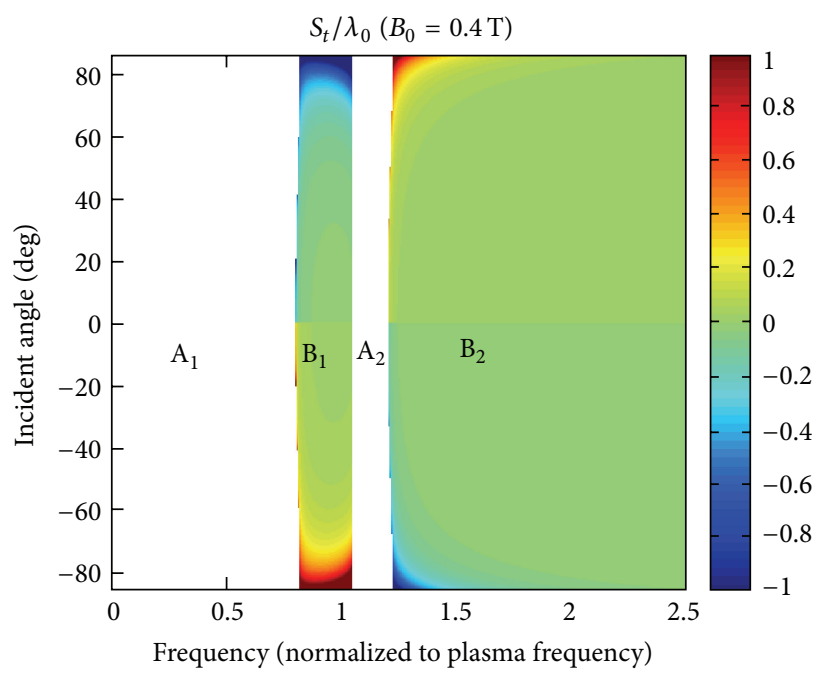

(d)

Figure 3: Goos-Hänchen lateral displacements of TM waves transmitted and reflected from the interface of vacuum and gyroelectric medium. The frequencies are normalized to $\omega_{p}$, and the displacements are normalized to $\lambda_{0}=2 \pi c / \omega$, the wavelength in the vacuum. (a) The lateral displacement of reflected waves $S_{r}$ with no applied magnetic field. (b) The lateral displacement of transmitted waves $S_{t}$ with no applied magnetic field. (c) The lateral displacement of reflected waves $S_{r}$ with an applied magnetic field $\bar{B}_{0}=+\widehat{z} 0.4 \mathrm{~T}$, and the Brewster angle is also shown (in white dashed line). (d) The lateral displacement of transmitted waves $S_{t}$ with the same applied magnetic field as (c).

when the incident wave comes from the lower left (as shown in Figure 1) and to be negative when the incident wave comes from the upper left. The displacements are normalized to $\lambda_{0}=$ $2 \pi c / \omega$, the wavelength in the vacuum.

For the plasma, when there is no applied magnetic field, since both $\varepsilon_{g}$ and $\tau$ are zero, the medium can be considered as a dispersive isotropic medium. Corresponding to the change of permittivity $\varepsilon_{V}$, there are two regions (A and B) in Figures 3(a) and 3(b). In region A, total reflection occurs at the interface. Thus, there is GH effect, and the lateral displacement of the reflected wave is not zero. But the transmitted wave is evanescent in the $x$ direction, making GH lateral displacement for transmitted waves meaningless, leaving a blank in
Figure 3(b). In region B, there is no total reflection and no GH lateral displacement for either reflected or transmitted waves. This is consistent with the fact of isotropic media case.

For TM waves, the existence of the applied magnetic field splits each region up into two, marked with subscript 1 and 2, shown in Figures $3(\mathrm{c})$ and $3(\mathrm{~d})$. In regions $A_{1}$ and $A_{2}$, total reflection occurs at the interface. In regions $B_{1}$ and $B_{2}$, although there is no total reflection at the interface, $\mathrm{GH}$ lateral displacements for both reflection and transmission are not zero, which is different from the isotropic media case.

It is also of interest to note that in regions $B_{1}$ and $B_{2}$ of Figure 3(c), there is a large displacement of reflected TM waves. It is due to the Brewster angle, shown in the white 


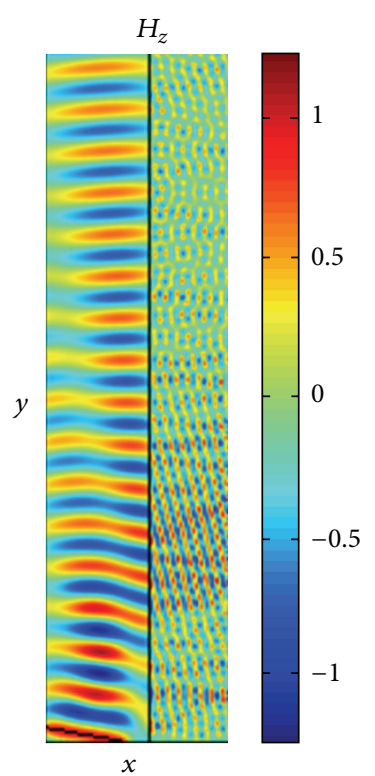

(a)

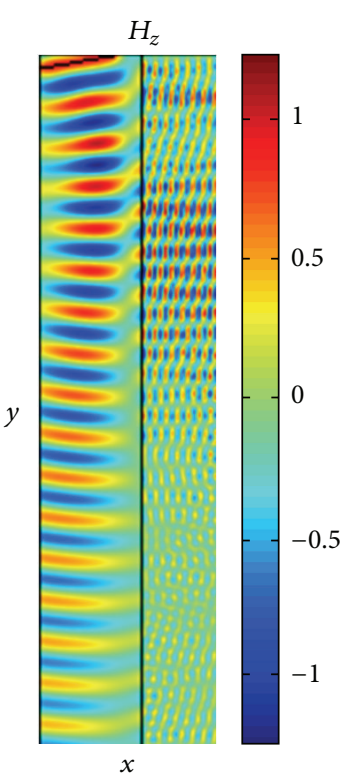

(b)

FIGURE 4: The reflection and transmission of a Gaussian TM beam incident from the vacuum to gyroelectric medium. The frequencies are both $\omega_{p}$, and the absolute values of the incident angles are both $80^{\circ}$. All the parameters are the same except the sign of incident angle. (a) The wave is incident from the lower left $\left(\theta_{i}=+80^{\circ}\right)$; (b) The wave is incident from the upper left $\left(\theta_{i}=-80^{\circ}\right)$.

dashed line in the figure. For TM waves, there is an abrupt phase change near the Brewster angle [41] which causes a large lateral displacement for the reflected beam. However, there is no abrupt change for the phase of the transmission coefficient at the Brewster angle. Therefore, it is reasonable to expect a large lateral displacement of the reflected beam near the Brewster angle but no such phenomenon for the transmitted beam.

It is important to note that the displacements with applied magnetic field are asymmetric with respect to the incident angle $\theta_{i}$ (see Figures 3(c) and 3(d)), but they are symmetric without applied magnetic field (see Figures 3(a) and 3(b)). For example, in region $\mathrm{B}_{2}, S_{r}$ is positive for a positive incident angle while it becomes negative for a negative incident angle. This asymmetric phenomenon is corresponding to the nonreciprocal property of the gyroelectric medium. If we only change the sign of incident angle $\theta_{i}$ from positive to negative, that is, the wave is incident from the upper left instead of the lower left, the field in gyroelectric medium changes even for the same absolute value of incident angle. Thus, the reflection and transmission coefficients change and so do the GH lateral displacements.

We show the reflection and transmission of a Gaussian TM beam incident from the vacuum to gyroelectric medium in Figure 4, which is the numerical result based on the finiteelement method. All the parameters used to calculate are the same except the sign of the incident angle. To be specific, $\omega=$ $\omega_{p}$ and $\left|\theta_{i}\right|=80^{\circ}$. The only difference is that in Figure 4(a), the TM wave incidents from the lower left and $\theta_{i}$ are positive, while in Figure 4(b), the wave comes from the upper left, and $\theta_{i}$ is negative. From Figure 4, we can see that the fields are different and so are the lateral displacements, which are consistent with our analysis as shown in Figure 3.

\section{Conclusion}

This paper investigates the Goos-Hänchen lateral displacements of a TM beam propagating at the interface between an isotropic medium and a gyroelectric medium in Voigt configuration. As different from the isotropic case, there are always GH effects, not only when total reflection occurs at the boundary but also when there is no total reflection. Furthermore, due to the nonreciprocal property of the gyroelectric medium, the sign of the incident angle also influences the displacements. The results here may have potential applications in magnetic modulations and Terahertz researches.

\section{Acknowledgments}

This work is sponsored by National Natural Science Foundation of China (51107003) and Beijing Jiaotong University (2009JBM092, 2011RC049, and 2011JBM110).

\section{References}

[1] M. S. Kushwaha and P. Halevi, "Magnetoplasmons in thin films in the Voigt configuration," Physical Review B, vol. 36, no. 11, pp. 5960-5967, 1987.

[2] M. S. Kushwaha and P. Halevi, "Magnetoplasma modes in thin films in the Faraday configuration," Physical Review B, vol. 35, no. 8, pp. 3879-3889, 1987.

[3] M. S. Kushwaha and P. Halevi, "Magnetoplasmons in thin films in the perpendicular configuration," Physical Review B, vol. 38, no. 17, pp. 12428-12435, 1988.

[4] J. R. Gillies and P. Hlawiczka, “TE and TM modes in gyrotropic waveguides," Journal of Physics D, vol. 9, no. 9, Article ID 1315, 1976. 
[5] J. R. Gillies and P. Hlawiczka, "Elliptically polarized modes in gyrotropic waveguides. II. An alternative treatment of the longitudinally magnetized case," Journal of Physics D, vol. 10, no. 14, Article ID 1891, 1977.

[6] P. Hlawiczka, "Elliptically polarized modes in gyrotropic waveguides," Journal of Physics D, vol. 9, no. 14, Article ID 1957, 1976.

[7] P. Hlawiczka, "A gyrotropic waveguide with dielectric boundaries: the longitudinally magnetised case," Journal of Physics D, vol. 11, no. 8, Article ID 1157, 1978.

[8] P. Hlawiczka, "The gyrotropic waveguide with a normal applied DC field," Journal of Physics D, vol. 11, no. 14, Article ID 1941, 1978.

[9] A. Eroglu and J. K. Lee, "Wave propagation and dispersion characteristics for a nonreciprocal electrically gyrotropic medium," Progress in Electromagnetics Research, vol. 62, pp. 237-260, 2006.

[10] L. W. Li, N. H. Lim, and J. A. Kong, "Cylindrical vector wave function representation of Green's dyadic in gyrotropic bianisotropic media," Journal of Electromagnetic Waves and Applications, vol. 17, no. 11, pp. 1589-1591, 2003.

[11] L. W. Li, N. H. Lim, W. Y. Yin, and J. A. Kong, "Eigenfunctional expansion of dyadic Green's functions in gyrotropic media using cylindrical vector wave functions," Journal of Electromagnetic Waves and Applications, vol. 17, no. 12, pp. 1731-1733, 2003.

[12] S. T. Ivanov and N. I. Nikolaev, "Magnetic-field effect on wave dispersion in a free semiconductor plasma slab," Journal of Physics $D$, vol. 32, no. 4, article 430, 1999.

[13] A. Boardman, N. King, Y. Rapoport, and L. Velasco, "Gyrotropic impact upon negatively refracting surfaces," New Journal of Physics, vol. 7, article 191, 2005.

[14] H. Huang, Y. Fan, F. Kong, B. I. Wu, and J. A. Kong, "Influence of external magnetic field on a symmetrical gyrotropic slab in terms of Goos-Hänchen shifts," Progress in Electromagnetics Research, vol. 82, pp. 137-150, 2008.

[15] H. Huang, Y. Fan, B. Wu, F. Kong, and J. A. Kong, "Surface modes at the interfaces between isotropic media and uniaxial plasma," Progress in Electromagnetics Research, vol. 76, pp. 1-14, 2007.

[16] F. Goos and H. Hänchen, "Ein neuer und fundamentaler Versuch zur Totalreflexion," Annals of Physics, vol. 1, pp. 333-334, 1947.

[17] F. Goos and H. Hänchen, "Neumessung des Strahlversetzungseffeketes bei Totalreflexion," Annals of Physics, vol. 5, pp. 251252, 1949.

[18] K. Artmann, "Berechnung der Seitenversetzung des totalreflektierten Strahles," Annals of Physics, vol. 2, pp. 87-102, 1948.

[19] R. H. Renard, "Total reflection: a new evaluation of the GoosHänchen shift," Journal of the Optical Society of America, vol. 54, no. 10, pp. 1190-1196, 1964.

[20] B. R. Horowitz and T. Tamir, "Lateral displacement of a light beam at a dielectric interface," Journal of the Optical Society of America, vol. 61, no. 5, pp. 586-594, 1971.

[21] F. Bretenaker, A. Le Floch, and L. Dutriaux, "Direct measurement of the optical Goos-Hänchen effect in lasers," Physical Review Letters, vol. 68, no. 7, pp. 931-933, 1992.

[22] O. Emile, T. Galstyan, A. Le Floch, and F. Bretenaker, "Measurement of the nonlinear Goos-Hänchen effect for Gaussian optical beams," Physical Review Letters, vol. 75, no. 8, pp. 15111513, 1995.

[23] B. M. Jost, A. A. R. Al-Rashed, and B. E. A. Saleh, "Observation of the Goos-Hänchen effect in a phase-conjugate mirror," Physical Review Letters, vol. 81, no. 11, pp. 2233-2235, 1998.
[24] P. Mazur and B. Djafari-Rouhani, "Effect of surface polaritons on the lateral displacement of a light beam at a dielectric interface," Physical Review B, vol. 30, no. 11, pp. 6759-6762, 1984.

[25] L. G. Wang, H. Chen, N. H. Liu, and S. Y. Zhu, "Negative and positive lateral shift of a light beam reflected from a grounded slab," Optics Letters, vol. 31, no. 8, pp. 1124-1126, 2006.

[26] C. F. Li, "Negative lateral shift of a light beam transmitted through a dielectric slab and interaction of boundary effects," Physical Review Letters, vol. 91, no. 13, Article ID 1339031, 4 pages, 2003.

[27] M. Merano, A. Aiello, C. W. Hooft, M. P. van Exter, E. R. Eliel, and J. P. Woerdman, "Observation of Goos-Hänchen shifts in metallic reflection," Optics Express, vol. 15, no. 24, pp. 1592815934, 2007.

[28] D. J. Hoppe and Y. Rahmat-Samii, "Gaussian beam reflection at a dielectric-chiral interface," Journal of Electromagnetic Waves and Applications, vol. 6, pp. 603-624, 1992.

[29] R. A. Depine and N. E. Bonomo, "Goos-Hänchen lateral shift for Gaussian beams reflected at achiral-chiral interfaces," Optik, vol. 103, no. 1, pp. 37-41, 1996.

[30] F. Wang and A. Lakhtakia, "Lateral shifts of optical beams on reflection by slanted chiral sculptured thin films," Optics Communications, vol. 235, no. 1-3, pp. 107-132, 2004.

[31] W. T. Dong, L. Gao, and C. W. Qiu, "Goos-Hänchen shift at the surface of chiral negative refractive media," Progress in Electromagnetics Research, vol. 90, pp. 255-268, 2009.

[32] W. J. Wild and C. L. Giles, "Goos-Hänchen shifts from absorbing media," Physical Review A, vol. 25, no. 4, pp. 2099-2101, 1982.

[33] E. Pfleghaar, A. Marseille, and A. Weis, "Quantitative investigation of the effect of resonant absorbers on the Goos-Hänchen shift," Physical Review Letters, vol. 70, no. 15, pp. 2281-2284, 1993.

[34] B. Zhao and L. Gao, "Temperature-dependent Goos-Hänchen shift on the interface of metal/dielectric composites," Optics Express, vol. 17, no. 24, pp. 21433-21441, 2009.

[35] P. R. Berman, "Goos-Hänchen shift in negatively refractive media," Physical Review E, vol. 66, no. 6, Article ID 067603, 3 pages, 2002.

[36] J. A. Kong, B. I. Wu, and Y. Zhang, "Lateral displacement of a Gaussian beam reflected from a grounded slab with negative permittivity and permeability," Applied Physics Letters, vol. 80, no. 12, pp. 2084-2086, 2002.

[37] A. Lakhtakia, "On planewave remittances and Goos-Hänchen shifts of planar slabs with negative real permittivity and permeability," Electromagnetics, vol. 23, no. 1, pp. 71-75, 2003.

[38] I. V. Shadrivov, A. A. Zharov, and Y. S. Kivshar, "Giant GoosHänchen effect at the reflection from left-handed metamaterials," Applied Physics Letters, vol. 83, no. 13, pp. 2713-2715, 2003.

[39] L. Remer, E. Mohler, W. Grill et al., "Nonreciprocity in the optical reflection of magnetoplasmas," Physical Review B, vol. 30, no. 6, pp. 3277-3282, 1984.

[40] J. J. Brion, R. F. Wallis, A. Hartstein, and E. Burstein, “Theory of surface magnetoplasmons in semiconductors," Physical Review Letters, vol. 28, no. 22, pp. 1455-1458, 1972.

[41] H. Huang, Y. Fan, B. I. Wu, and J. A. Kong, "Positively and negatively large Goos-Hänchen lateral displacements from a symmetric gyrotropic slab," Applied Physics A, vol. 94, no. 4, pp. 917922, 2009.

[42] M. S. Kushwaha, "Plasmons and magnetoplasmons in semiconductor heterostructures," Surface Science Reports, vol. 41, no. 18, pp. 1-416, 2001. 

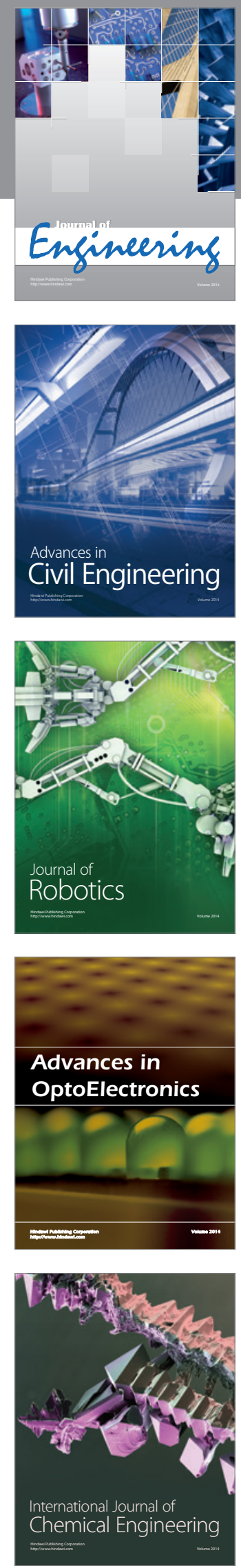

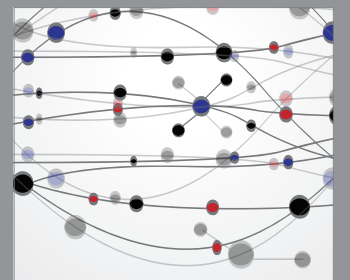

The Scientific World Journal
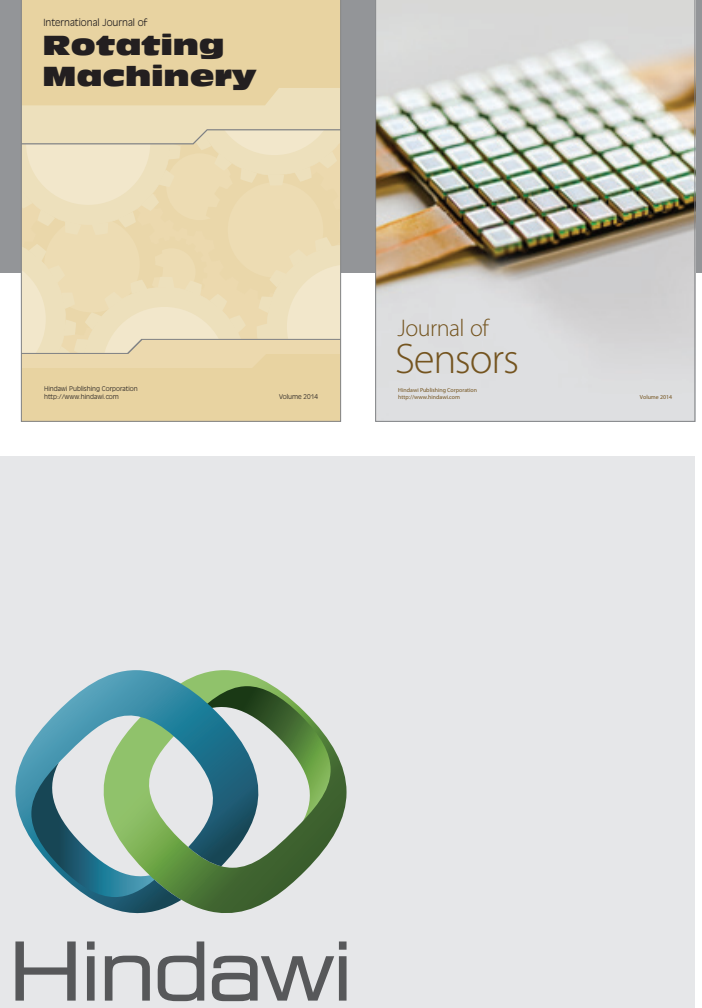

Submit your manuscripts at http://www.hindawi.com
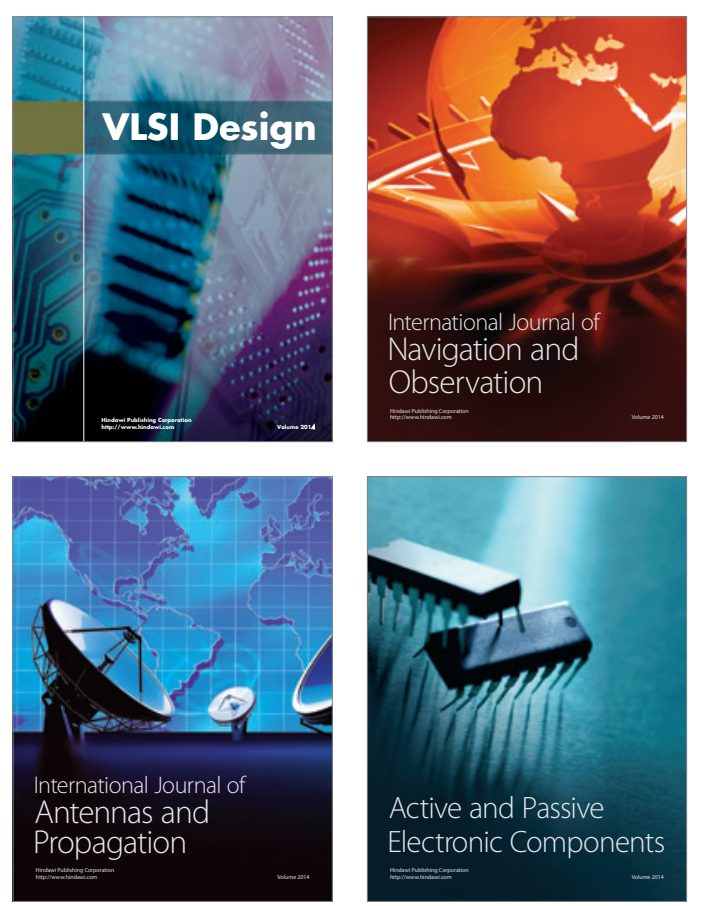
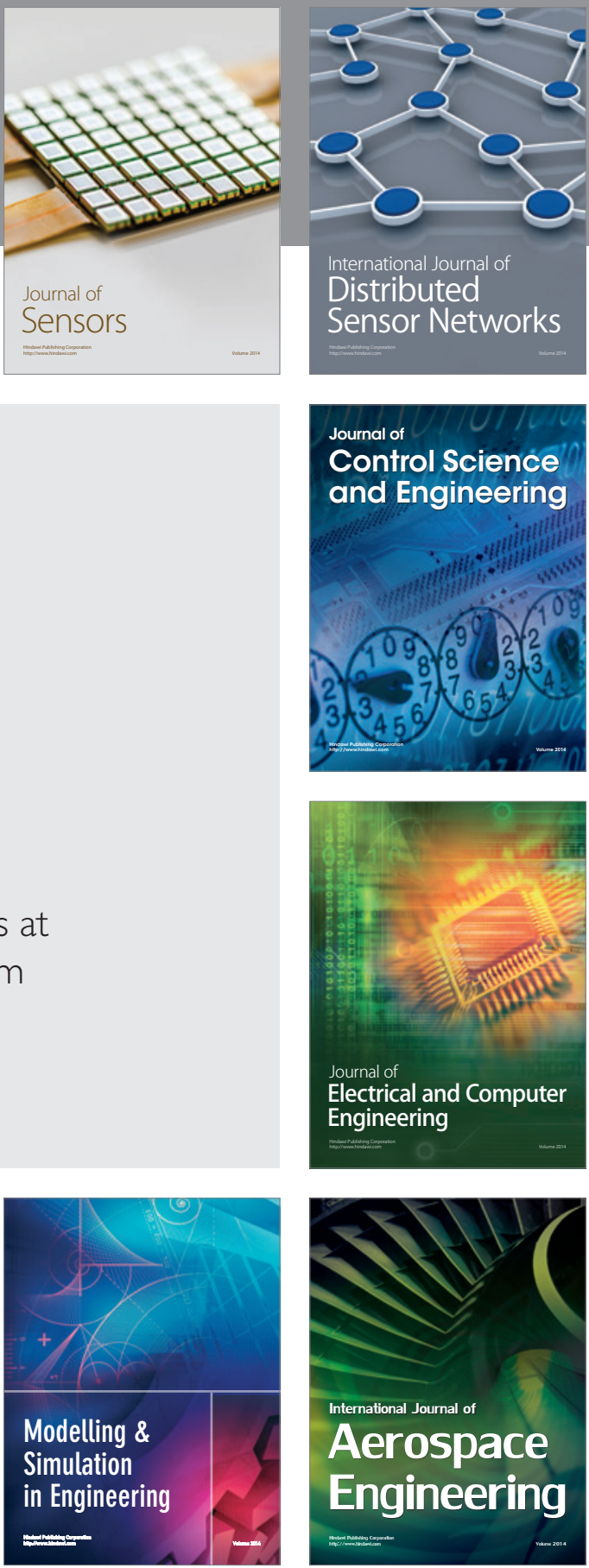

Journal of

Control Science

and Engineering
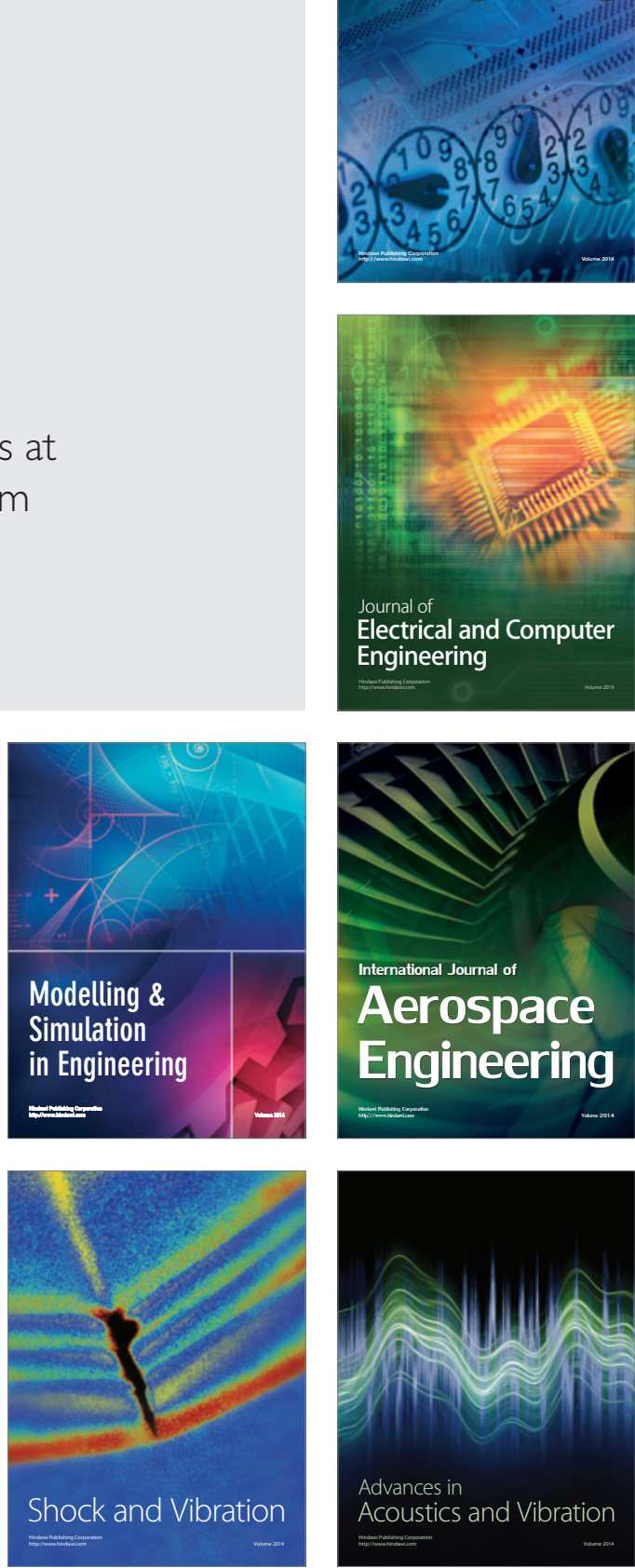